\title{
Centrifuge Model Tests of the Effect of the Ground Motion Coherence Function on the Amplification of a Saturated Soil- Water Site
}

\author{
Xijun Song, ${ }^{1}$ Juan Liu, ${ }^{2}$ Jingyan Lan $\mathbb{D}^{1},{ }^{1}$ and Ting Wang ${ }^{1}$ \\ ${ }^{1}$ Guangxi Key Laboratory of Geomechanics and Geotechnical Engineering, Guilin University of Technology, Guilin 541004, China \\ ${ }^{2}$ Institute of Engineering Mechanics, China Earthquake Administration, Harbin 150080, China
}

Correspondence should be addressed to Jingyan Lan; lanjy1999@163.com

Received 3 February 2021; Revised 21 April 2021; Accepted 5 May 2021; Published 15 May 2021

Academic Editor: Constantinos Loupasakis

Copyright (C) 2021 Xijun Song et al. This is an open access article distributed under the Creative Commons Attribution License, which permits unrestricted use, distribution, and reproduction in any medium, provided the original work is properly cited.

\begin{abstract}
Two sets of dynamic centrifugal model tests were designed and implemented in this study: the overlying waterless surface and the water-covered surface. Based on the use of the El Centro waves with different intensities as the base input, the seismic time history at the surface of two sets of free site models was obtained. According to the results of the site response at two sets of the free site surface obtained with a traditional spectral ratio, the coherence functions at the surface and the base were used to modify the traditional spectral ratio for analysis and to evaluate the effect of the ground motion coherence function for site amplification. The modal characteristics and the amplification effect of a typical saturated soil water free site were summarized at the same time. The results showed that the ground response results of the two groups of typical free site centrifugal models were greatly influenced by the coherence function. In the low frequency phase, the coherence function of the amplification spectrum of the site response decreased significantly, while in the high frequency phase, the decrease trend decreased. The coherence function had a significant effect on the first-order mode of the free site. The first-order mode frequency and the amplification factor of a typical free site could be identified effectively. Compared with the saturated land free site model, the saturated soil water free site model had higher-order modes due to the overlying water. It was shown that the overlying water, as part of a complex medium system, could be ignored in the site response and basic cycle estimation.
\end{abstract}

\section{Introduction}

The coherence function is a physical quantity that describes the statistical characteristics of the correlation between two points in the ground motion space, and it can reflect the relationship between the correlation of each frequency component of the ground motion and the frequency and the distance between the two points. There have been many previous studies on the influencing factors of the ground motion coherence function $[1,2]$. Chen and Li [3] and Ding and Song [4] used numerical analysis methods to determine that the geological structure of a site had a greater influence on the coherence function. Wang et al. [5] and others analyzed the seismic records of the California Strong Motion Instrumentation Program (CSMIP), and they showed that the coherence of the ground motion decreased with the increase of the epicenter distance and the depth from the surface, and the coherence of the ground motion had a greater impact on the low frequency band of seismic waves. By analyzing the ground motion data of the Taiwan LSST array, Abrahamson et al. [6] found that the magnitude had less influence on the coherence function when the frequency was greater than $10 \mathrm{~Hz}$, while magnitude had a greater influence when the frequency was less than $10 \mathrm{~Hz}$. Steidl's [7] research showed that the coherence function played an important role and influenced the propagation law and attenuation characteristics of ground motions. There is a difference in the wave impedance at the free site layering of a multi-degree-of-freedom system. Therefore, seismic waves will produce refraction and reflection at a soil interface. The formation of the destructive interference of the upward wave site and the downward wave site causes the amplitude of the Fourier 
spectrum of the ground motion in a specific frequency band to be greatly reduced, forming a pseudoresonance spectrum hole [8]. In order to solve this problem, Wang [9] used the traditional spectral ratio method to calculate the site transfer function for the records of four small earthquakes obtained with the Treasure Island geotechnical array in San Francisco Bay, USA. Additionally, Wang [9] convolved the result with the relevant function to obtain a stable site amplification effect, thus eliminating the so-called pseudoresonance phenomenon.

At present, due to the lack of acquired marine seismic observation records, the research methods and conclusions of a sea ground motion simulation and the site amplification effect cannot be effectively verified [10-12]. There are few public reports on the effect of the coherence function of the ground motion on the site amplification of a complex saturated soil-water system. In recent years, because centrifugal model can truly reproduce the prototype stress state, more and more geotechnical problems can be solved by centrifugal model test, which is the mainstream and hot spot in the field of geotechnical seismic engineering. It is widely used in slope stability analysis, dynamic simulation of underground space and tunnel engineering, soil seismic liquefaction, and so on. Toboada-Urtuzuastegui et al. [13] carried out the centrifuge model test on the liquefied sand slope. Through the analysis of acceleration sensor and pore pressure sensor records, it shows that the expansion response of the slope will increase with the increase of the input seismic amplitude value, the slope deformation will accumulate, and the development of the water level to crowding displacement will be reduced. The centrifugal model test of tunnel engineering was carried out by Cilingir and Madabhushi [14]. By setting different tunnel cross-sections and geometric shapes, it is found that the bending moment and internal force increase with the increase of the maximum base acceleration input, and the stability of the circular tunnel is better than that of the square tunnel. Chen and Shen [15] designed two groups of square tunnel models with and without isolation layer by using the dynamic centrifuge test and explored the seismic effect of the isolation layer on the square tunnel by inputting different vibration loads. The results show that when the isolation layer is placed around the outside of the tunnel, the bending moment at the corner will be greatly reduced, thus improving the seismic performance of the tunnel. El shafee et al. [16] designed several centrifuge model tests to evaluate the effect of biaxial vibration on soil liquefaction in the horizontal site and compared with the results of uniaxial vibration. The results show that the commonly used method of increasing the uniaxial vibration by $10 \%$ does not represent the real site response, and the biaxial vibration can better reflect the problem of soil structure interaction.

In view of this, in this study, the centrifuge shaking table test was relied on to design two sets of free site homogeneous stratification models. These models could restore saturated soft soil land sites and saturated soft soil sea areas covered with water. The acquired ground motion record site response analysis was used, the difference between the vibration characteristics of the two free site models and the site response amplification spectrum was discussed, and the influence of the ground motion coherence function on the typical free site amplification was summarized and evaluated. The research results helped to improve and deepen the understanding of the site of marine soil dynamics and sea ground motion site effects. These results have certain reference value and engineering significance for improving the accuracy of marine engineering seismic design and reducing engineering seismic risks.

\section{Dynamic Centrifugal Model Test Scheme and Results}

In this centrifuge shaking table test, the TK-C500 geotechnical centrifuge test machine of the National Engineering Laboratory for Port Hydraulic Construction Technology, Tianjin Research Institute for Water Transport Engineering, was used. The maximum centrifugal acceleration was $250 \mathrm{~g}$, the maximum radius was $5 \mathrm{~m}$, and the maximum effective load was $5 \mathrm{t}$. The geotechnical centrifuge was equipped with an advanced shaking table system that could provide highprecision vertical/horizontal bidirectional vibration for a $100 \mathrm{~g}$ centrifugal state. The maximum vibration acceleration was $40 \mathrm{~g}$, the maximum duration was $3 \mathrm{~s}$, the maximum vibration frequency was $350 \mathrm{~Hz}$, and the design vibration effective load was $0.8 \mathrm{t}$. The comprehensive performance reached the international advanced level. The model box used in the experiment was a laminated ring shear model box made of light and high strength alloy aluminum that could effectively reduce the side boundary effect. The internal dimensions could meet the needs of many types of model tests.

In order to fully investigate the influence of overlying seawater on the ground motion, two sets of free site models were designed in this study. The purpose was to compare the difference of the measured seismic wave records between the two groups for saturated site conditions with and without water over the surface. In addition to the overlying water, the centrifugal similarity rate, model depth, and sensor layout of the two groups of models were consistent. Since the seawater depth in China's offshore waters was about $15 \mathrm{~m}$, considering the size of the shear model box, the model box was $1.00 \mathrm{~m}$ wide, the saturated soil was $0.30 \mathrm{~m}$ high, and the overlying water was $0.15 \mathrm{~m}$ high. Therefore, the centrifugal acceleration used in the test was $100 \mathrm{~g}$. The similarity rate was $n=100$, which could simulate a prototype offshore saturated water and soil site with an overlying water depth of $15 \mathrm{~m}$. The sensor layout is shown in Figure 1. The acceleration sensor A0 was regarded as the base acceleration, and A1 was regarded as the ground acceleration.

Input seismic wave loading form: The natural wave $\mathrm{El}$ Centro wave was used as the base input, and the input of the ground motion was controlled by adjusting the amplitude. For the above two models, the loading form followed the principle of small to large, with $0.025 \mathrm{~g}, 0.05 \mathrm{~g}, 0.10 \mathrm{~g}$, $0.20 \mathrm{~g}$, and $0.40 \mathrm{~g}$ loaded step by step.

According to the given design plan of the centrifugal model test and the form of ground motion loading, the two centrifugal models of the water-free surface and the watercovered surface were subjected to vibration tests, and the acceleration record results of the sensor A1 in the model 


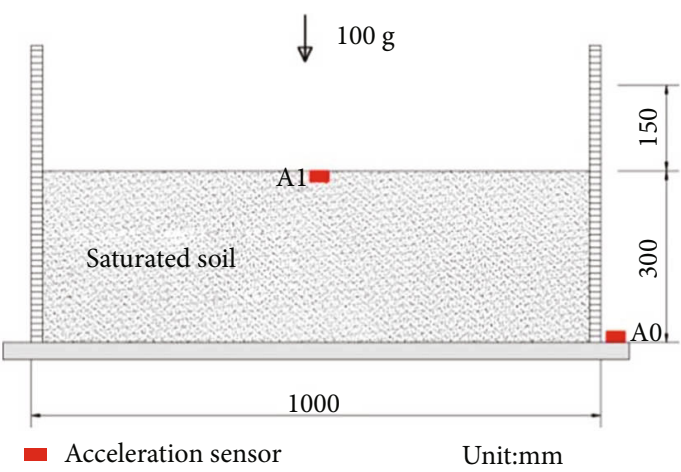

(a)

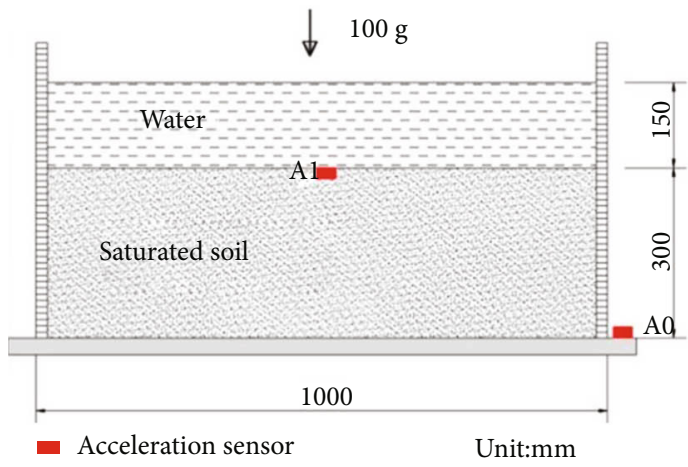

(b)

FIGURE 1: Design models of THE dynamic centrifuge shake table test: (a) Model 1 for the surface without water. (b) Model 2 for the surface with water. (units: $\mathrm{mm}$ ).

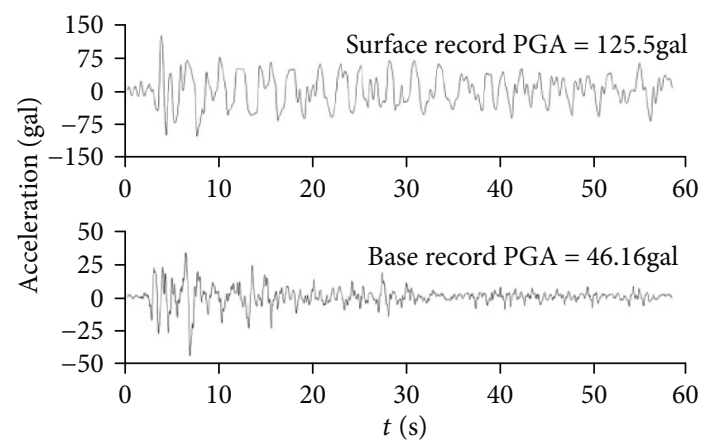

(a)

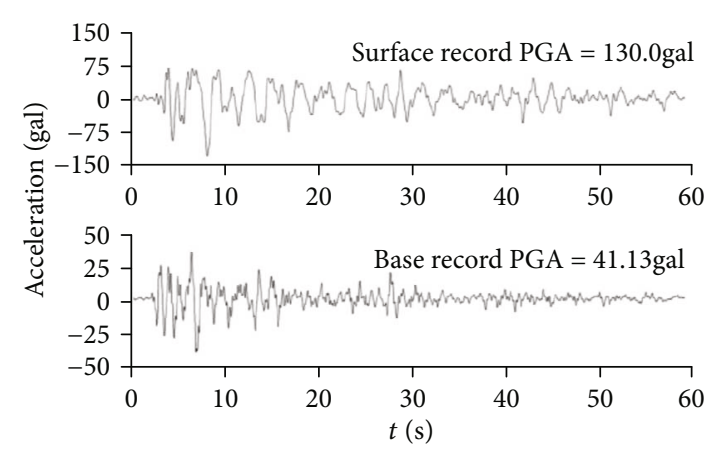

(b)

FIgURE 2: Acceleration time history response of EL-1 situation: (a) model 1 and (b) model 2.

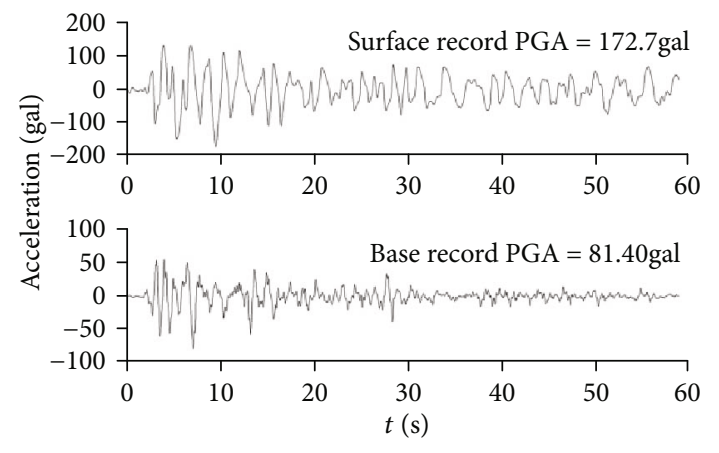

(a)

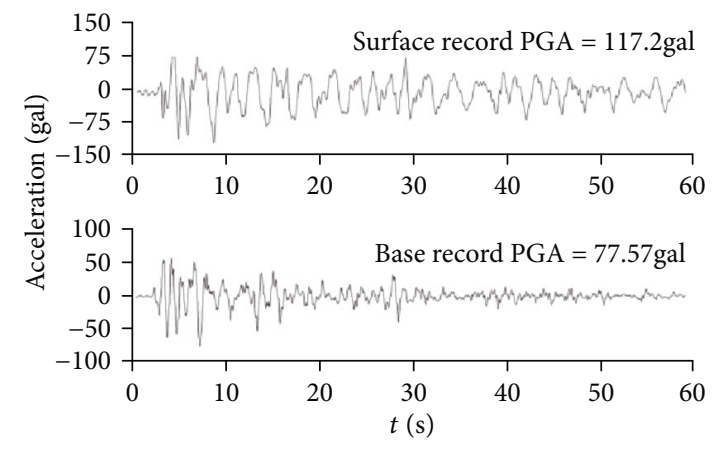

(b)

Figure 3: Acceleration time history response of EL-2 situation: (a) model 1 and (b) model 2.

box were given. The acceleration records of the five groups of dynamic centrifugal models (EL-1-EL-5) are shown in Figures 2-6.

\section{Site Response Analysis Method}

From the point of view of the system, the ground motion was a system composed of the source effect, path effect, site effect, instrument response, and other physical processes. In the frequency domain, the Fourier spectrum $A\left(M_{\mathrm{o}}, R, f\right)$ of the site ground motion could be expressed as four physical processes: source effect $(S)$, path effect $(P)$, site effect $(G)$, and instrument response $(I)$, the product of which was

$$
A\left(M_{o}, R, f\right)=S\left(M_{o}, f\right) P(R, f) G(f) I(f),
$$

where $M_{\mathrm{o}}$ is the moment magnitude, $R$ is the hypocentral distance, and $f$ is the frequency. 


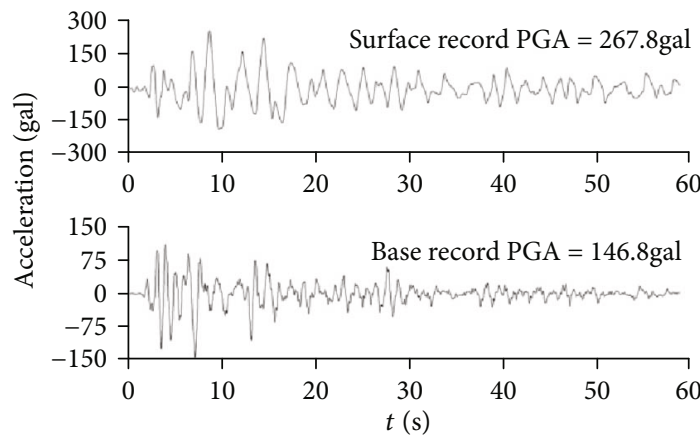

(a)

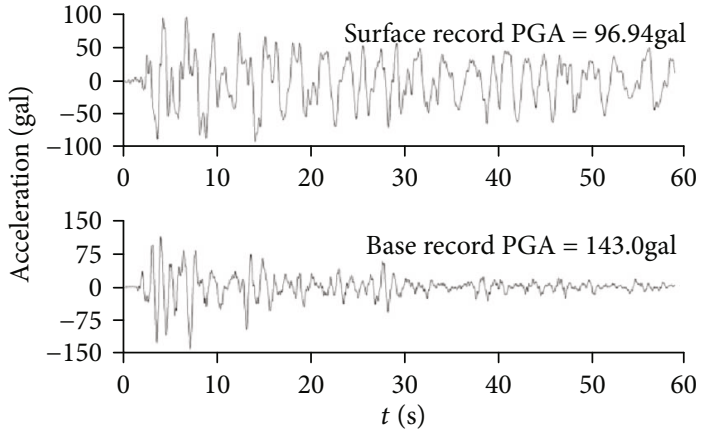

(b)

FIgURE 4: Acceleration time history response of EL-3 situation: (a) model 1 and (b) model 2.

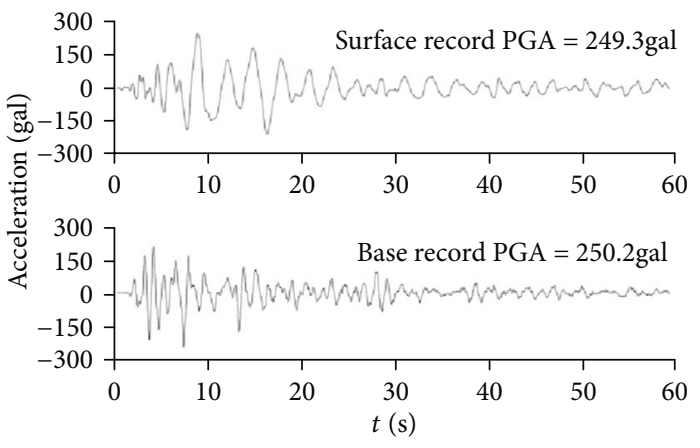

(a)

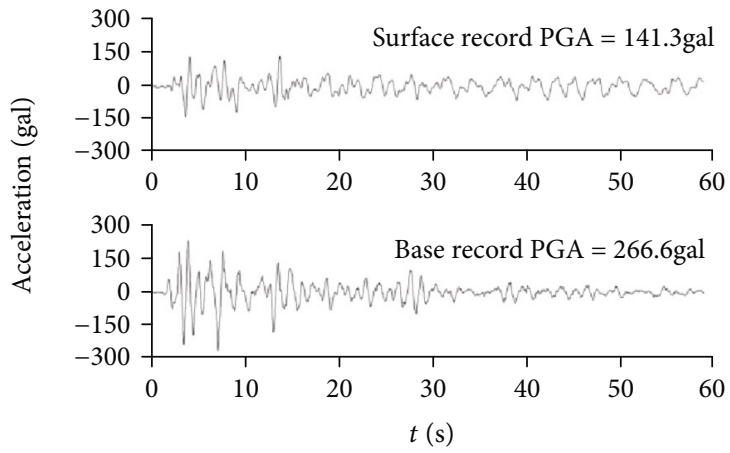

(b)

Figure 5: Acceleration time history response of EL-4 situation: (a) model 1 and (b) model 2.

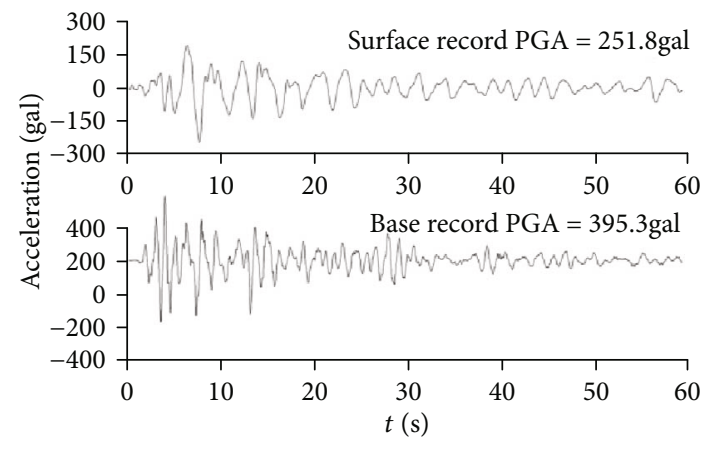

(a)

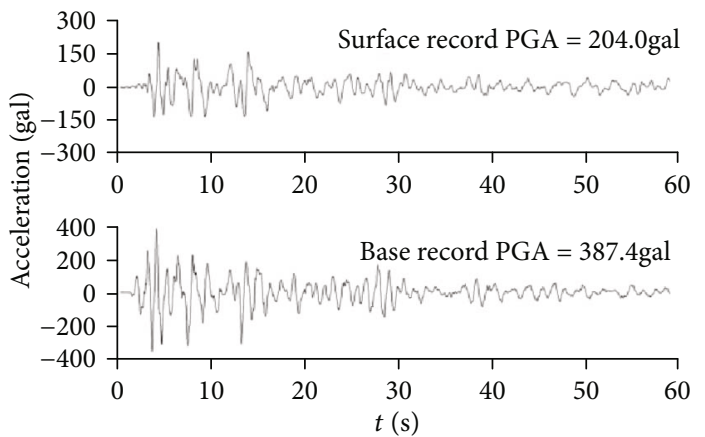

(b)

Figure 6: Acceleration time history response of EL-5 situation: (a) model 1 and (b) model 2.

The traditional spectral ratio method was proposed by Borcherdt [17]. A hard rock site was selected as a reference site to calculate the ratio of the Fourier spectrum of the ground motion records between the soil layer site and the rock site. The source effect and the path effect in the ground motion record were eliminated in order to obtain an estimate of the site response. The soil layer site transfer function was

$$
S_{T}=\frac{S_{H S}}{S_{H R}}
$$

where $S_{H S}$ is the Fourier spectrum of the horizontal ground motion of the soil layer site, and $S_{H R}$ is a reference to the Fourier spectrum of the ground motion horizontally.

In this study, the uphole/downhole spectral ratio method was adopted; that is, the ground motion records at different depths had the same source, path effect, and instrument response in the same geotechnical array. Therefore, the influences of the source, path effect, and instrument response could be eliminated by calculating the Fourier spectral ratio (traditional spectral ratio method) of the ground motion records at different depths with the underground bedrock as the reference site. Additionally, Steidl et al. [7] proposed 


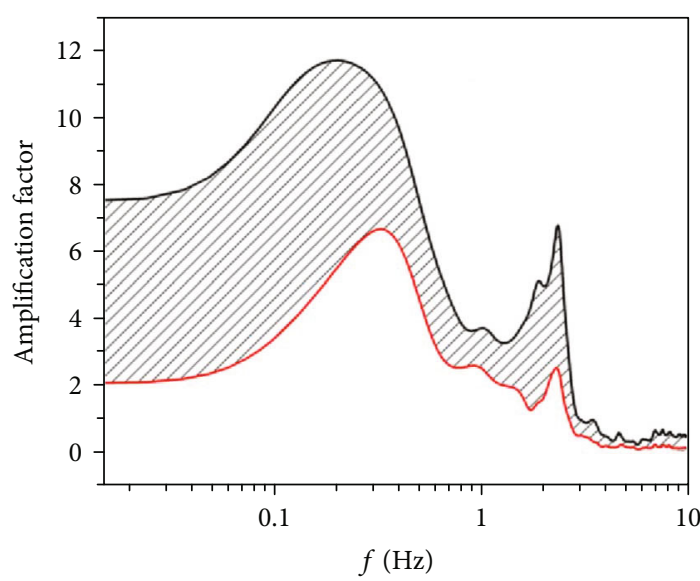

- Unmultiplied coherent function

- Multiplied coherence function

(a)

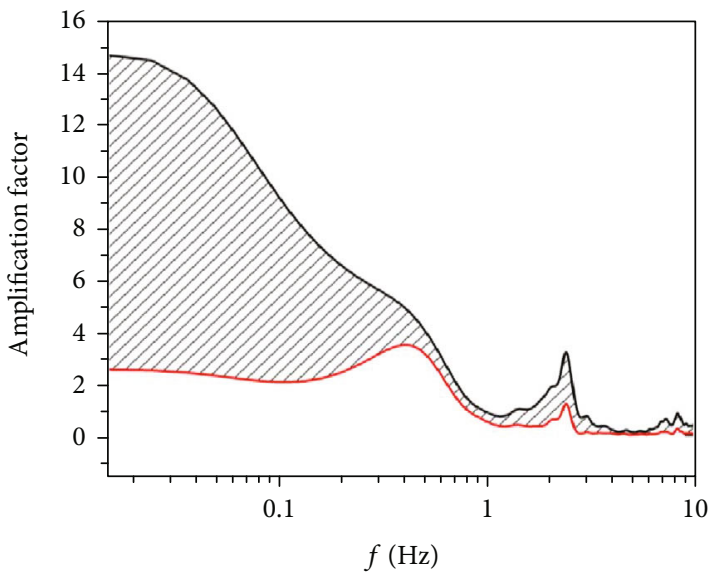

- Unmultiplied coherent function

_ Multiplied coherence function

(c)

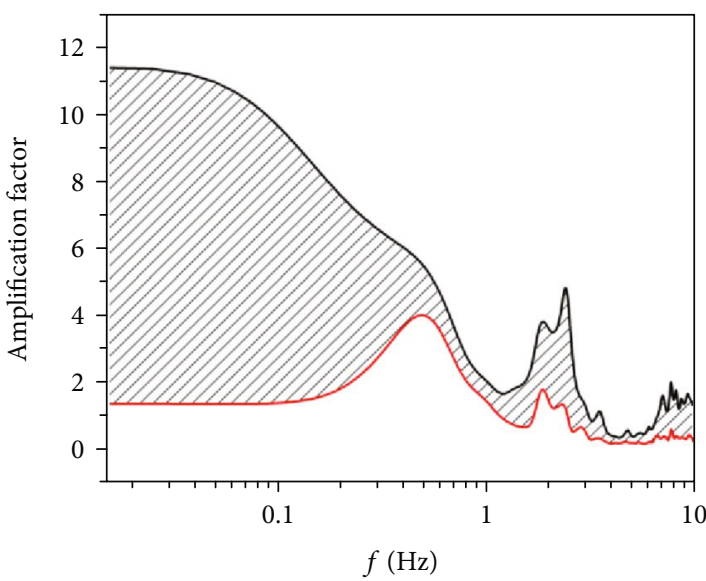

Unmultiplied coherent function Multiplied coherence function

(b)

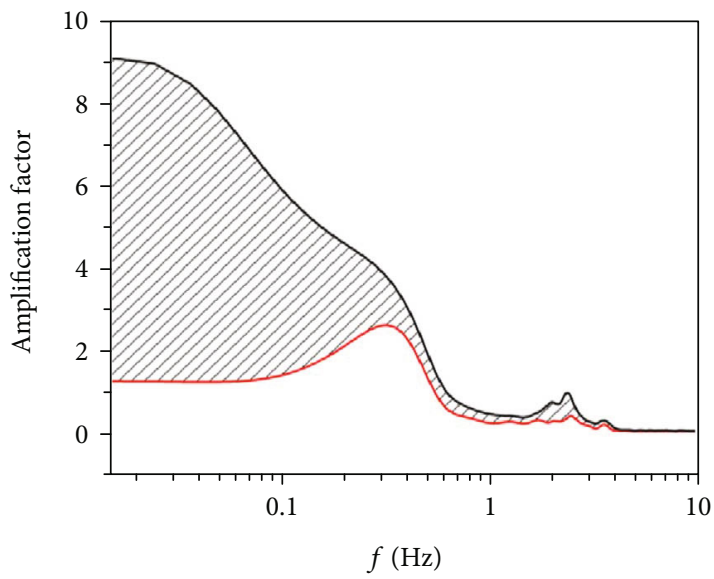

- Unmultiplied coherent function

_ Multiplied coherence function

(d)

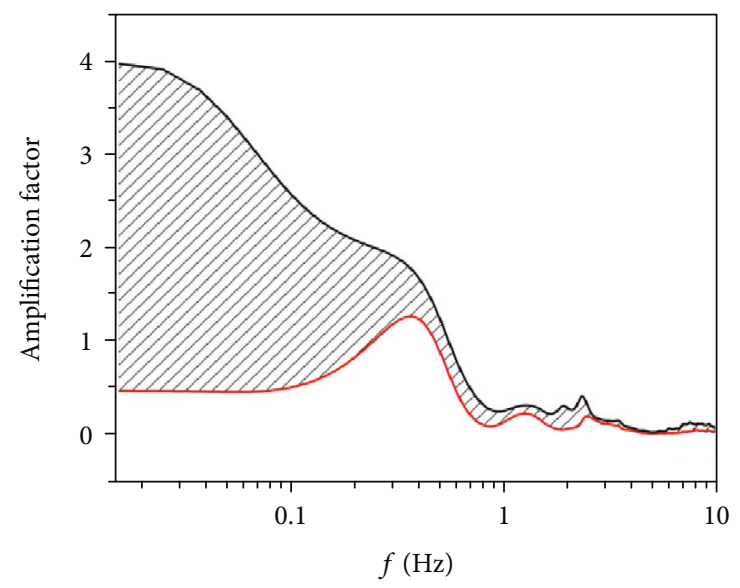

- Unmultiplied coherent function

Multiplied coherence function

(e)

Figure 7: Amplified factor of the site response of the unmultiplied coherent function and the multiplied coherent function (model 1 for surface without water): (a) EL-1 situation, (b) EL-2 situation, (c) EL-3 situation, (d) EL-4 situation, and (e) EL-5 situation. 


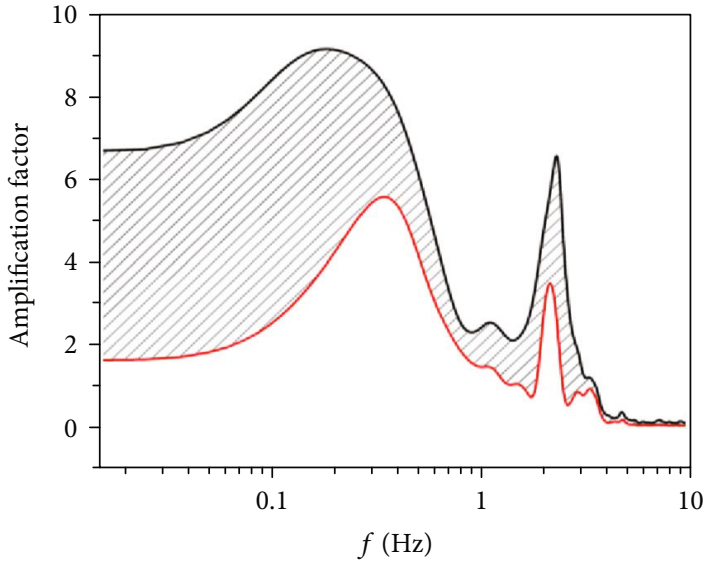

- Unmultiplied coherent function

_ Multiplied coherence function

(a)

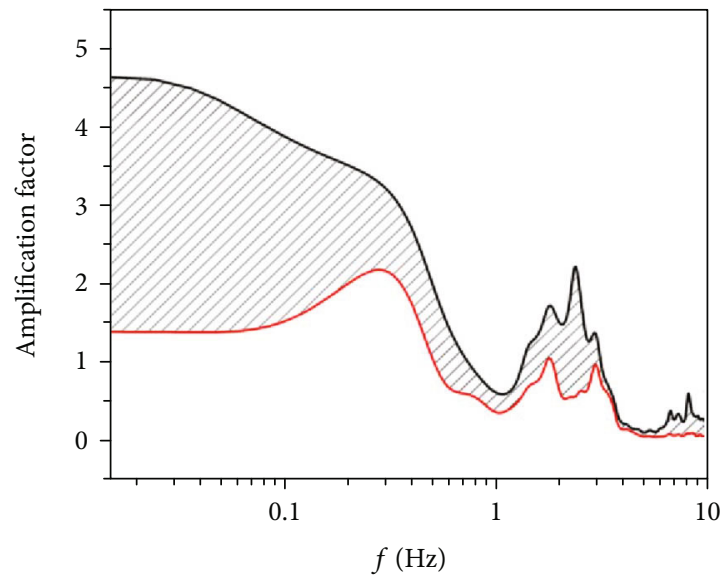

Unmultiplied coherent function

Multiplied coherence function

(c)

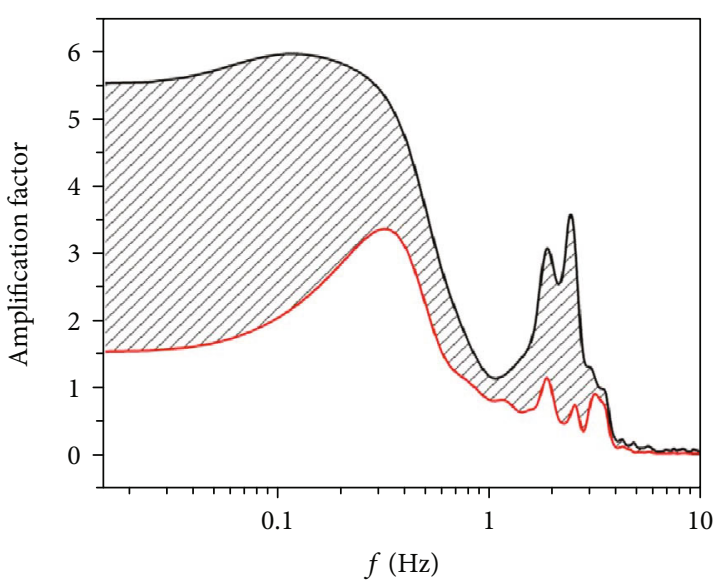

- Unmultiplied coherent function

- Multiplied coherence function

(b)

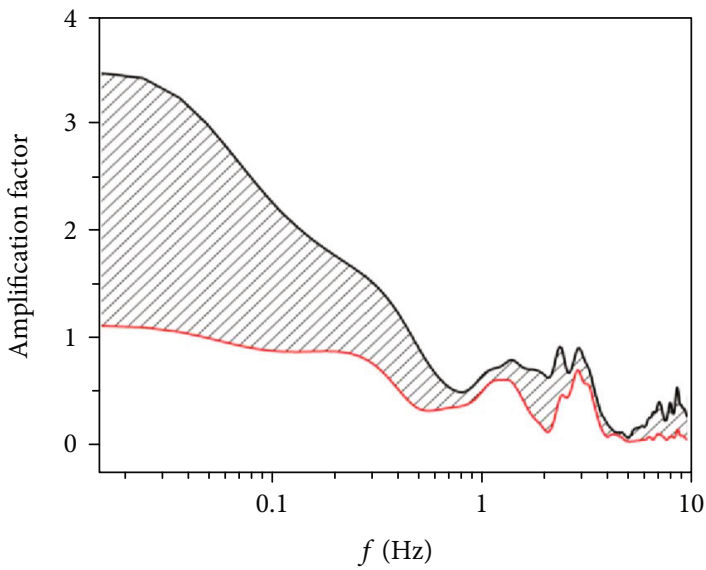

Unmultiplied coherent function Multiplied coherence function

(d)

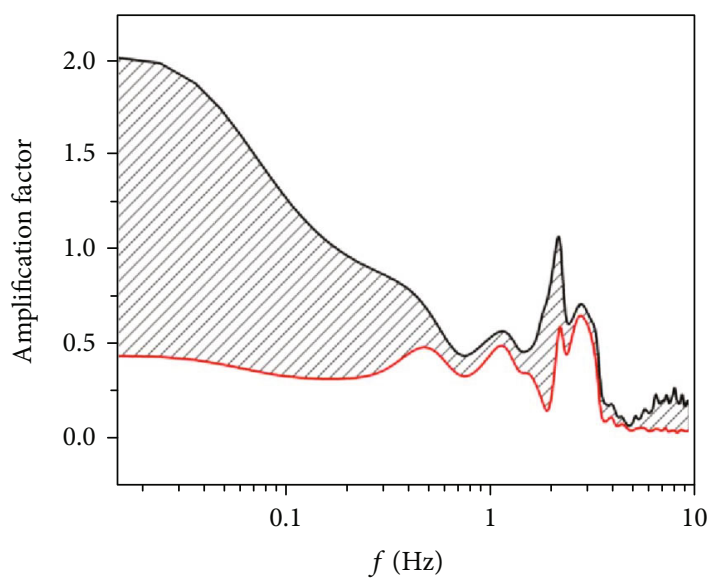

- Unmultiplied coherent function

- Multiplied coherence function

(e)

Figure 8: Amplified spectrum of the site response of the unmultiplied coherent function and multiplied coherent function (Model for the surface with water): (a) EL-1 situation, (b) EL-2 situation, (c) EL-3 situation, (d) EL-4 situation, and (e) EL-5 situation. 
Table 1: Amplification factor and frequency of different vibration modes loaded with different conditions for the model for the surface without water. $f_{d}$ : predominant frequency; AF: amplification factor.

\begin{tabular}{|c|c|c|c|c|c|c|c|c|}
\hline \multirow{3}{*}{ Input loading $(\mathrm{g})$} & \multicolumn{4}{|c|}{ Unmultiplied coherent function } & \multicolumn{4}{|c|}{ Multiplied coherent function } \\
\hline & \multicolumn{2}{|c|}{ First-order mode } & \multicolumn{2}{|c|}{$\begin{array}{c}\text { Second-order } \\
\text { mode }\end{array}$} & \multicolumn{2}{|c|}{ First-order mode } & \multicolumn{2}{|c|}{$\begin{array}{l}\text { Second-order } \\
\text { mode }\end{array}$} \\
\hline & $f_{d}$ & $\mathrm{AF}$ & $f_{d}$ & $\mathrm{AF}$ & $f_{d}$ & $\mathrm{AF}$ & $f_{d}$ & $\mathrm{AF}$ \\
\hline 0.025 & 0.21 & 11.75 & 2.39 & 6.78 & 0.33 & 6.67 & 2.36 & 2.50 \\
\hline 0.050 & 0.40 & 5.95 & 1.88 & 3.74 & 0.49 & 3.93 & 1.89 & 1.67 \\
\hline 0.100 & 0.34 & 5.37 & 2.42 & 3.21 & 0.40 & 3.49 & 2.42 & 1.21 \\
\hline 0.200 & 0.29 & 3.98 & 2.40 & 0.94 & 0.32 & 2.60 & 2.49 & 0.38 \\
\hline 0.400 & 0.29 & 1.93 & 1.26 & 0.31 & 0.37 & 1.27 & 1.25 & 0.22 \\
\hline
\end{tabular}

TABLE 2: Amplification factor and frequency of different vibration modes loaded with different conditions for the model for the surface with water. $f_{d}$ : predominant frequency; AF: amplification factor.

\begin{tabular}{|c|c|c|c|c|c|c|c|c|}
\hline \multirow{3}{*}{ Input loading (g) } & \multicolumn{4}{|c|}{ Unmultiplied coherent function } & \multicolumn{4}{|c|}{ Multiplied coherent function } \\
\hline & \multicolumn{2}{|c|}{ First-order mode } & \multicolumn{2}{|c|}{$\begin{array}{c}\text { Second-order } \\
\text { mode }\end{array}$} & \multicolumn{2}{|c|}{ First-order mode } & \multicolumn{2}{|c|}{$\begin{array}{l}\text { Second-order } \\
\text { mode }\end{array}$} \\
\hline & $f_{d}$ & $\mathrm{AF}$ & $f_{d}$ & $\mathrm{AF}$ & $f_{d}$ & $\mathrm{AF}$ & $f_{d}$ & $\mathrm{AF}$ \\
\hline 0.025 & 0.20 & 9.20 & 2.36 & 6.58 & 0.34 & 5.58 & 2.19 & 3.48 \\
\hline 0.050 & 0.12 & 5.98 & 1.88 & 3.07 & 0.32 & 3.37 & 1.87 & 1.14 \\
\hline 0.100 & 0.26 & 3.36 & 1.83 & 1.70 & 0.28 & 2.16 & 1.83 & 1.02 \\
\hline 0.200 & 0.21 & 1.75 & 1.42 & 0.78 & 0.18 & 0.86 & 1.33 & 0.60 \\
\hline 0.400 & 0.35 & 0.83 & 1.20 & 0.56 & 0.49 & 0.47 & 1.18 & 0.48 \\
\hline
\end{tabular}

a method of eliminating pseudoresonance by multiplying the coherence function by the transfer function and calculating a more reliable site response. The coherence function was calculated as follows:

$$
\gamma_{12}(f)=\frac{S_{12}(f)}{\sqrt{S_{11}(f) S_{22}(f)}}
$$

where $S_{11}(f)$ is the self-power spectrum of the ground motion records at a certain depth, $S_{22}(f)$ is the self-power spectrum of the ground motion records at the reference sites, and $S_{12}$ $(f)$ is the crosspower spectrum between the ground motion records at a given depth and those at the reference site.

In this study, the ground motion recorded by the strong seismometer A0 was used as the ground motion of the bedrock reference site, and the ground motion recorded by the strong seismometer A1 was used as the surface ground motion. The Fourier spectrum of the acceleration time history was calculated, and the smoothing was carried out by the Parzen window of $0.4 \mathrm{~Hz}$. Then, the Fourier spectrum ratio and coherence function of the ground surface were calculated. Finally, the Fourier spectrum ratio and the coherence function were multiplied to obtain a more reliable site response.

According to the above method, the acceleration time history data obtained with the centrifuge model test were used to calculate the site response of the surface model with water and the model without water model for different input loading, as well as the Fourier spectral ratio of the unmultiplied coherent function, namely, the amplified spectrum of the site response [18].

\section{Result Analysis}

The site response amplification spectra of the two groups of free site models with and without water were calculated using the above method, as shown in Figures 7 and 8, and the difference of the site response (shaded part) between the unmultiplied coherent function (black solid line) and the multiplied coherent function (red solid line) was compared.

It can be seen from Figure 7 that for the model without water, whether the site reaction was the unmultiplied coherent function or the multiplied coherent function, the amplification effect of the surface mainly occurred at the low frequency stage of less than $0.5 \mathrm{~Hz}$, and the amplification factor of the first-order mode was the largest. For the highfrequency components above $7 \mathrm{~Hz}$, the amplification factor tended towards zero, indicating that the site soil had no amplification effect within this frequency range. As the input load increased, the site response amplification factor decreased. In the low frequency stage of $0-0.5 \mathrm{~Hz}$, the difference between the unmultiplied coherence function and the multiplied coherent function was the largest. As shown in Table 1, the frequency of the first-order mode of the unmultiplied coherent function differed greatly from that of the multiplied coherent function, with the biggest differences as follows. When $0.025 \mathrm{~g}$ was loaded, the frequency of the first-order mode of the unmultiplied coherent function was $0.21 \mathrm{~Hz}$, while the frequency of the first-order mode of the multiplied coherent function was $0.33 \mathrm{~Hz}$, with a difference of $0.12 \mathrm{~Hz}$. However, the frequency difference between the second-order mode of the unmultiplied coherent function and that of the second-order mode of the multiplied coherent 


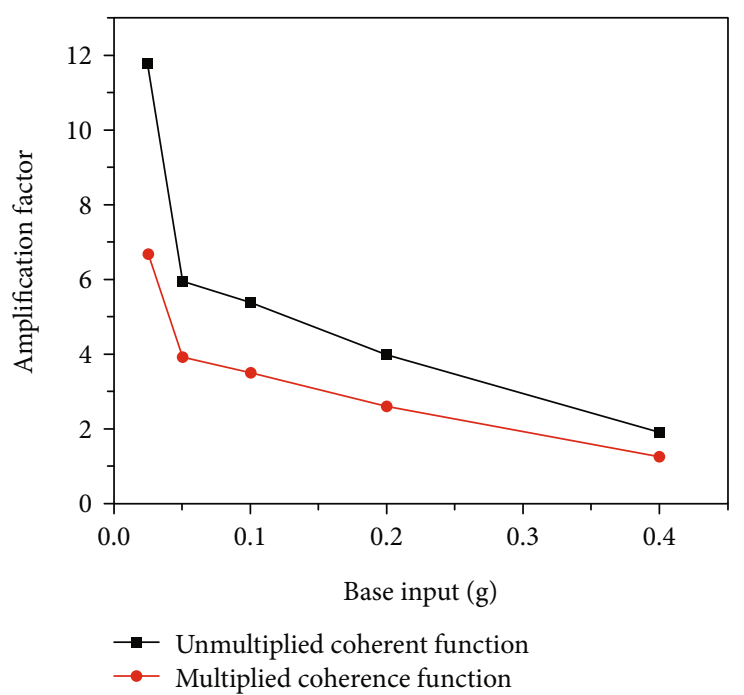

(a)

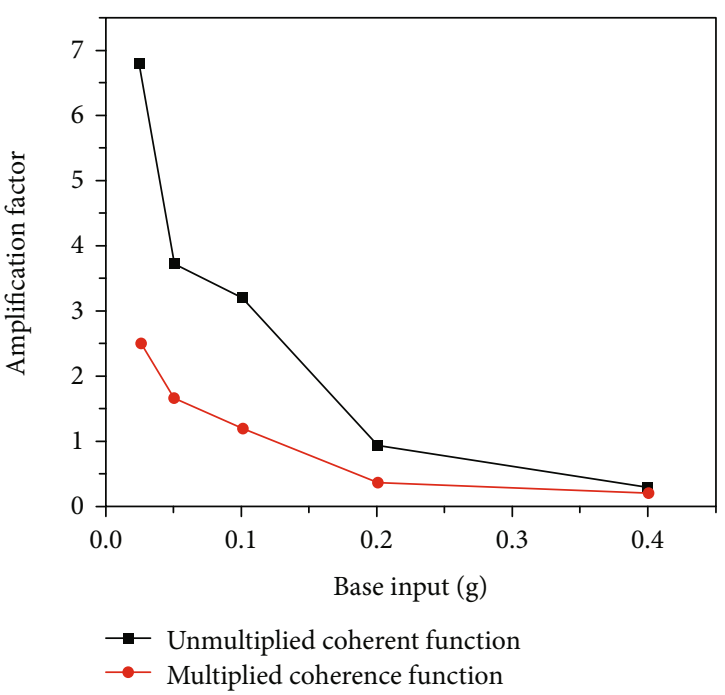

(b)

Figure 9: Amplification factor comparison chart of the unmultiplied coherent function and multiplied coherent function for different conditions (model 1 for the surface without water): (a) firs-order mode and (b) second-order mode.

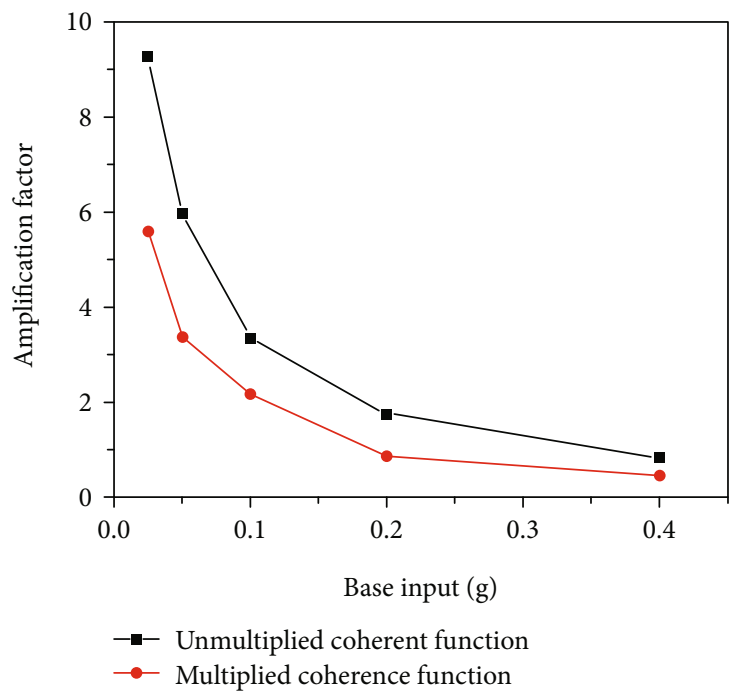

(a)

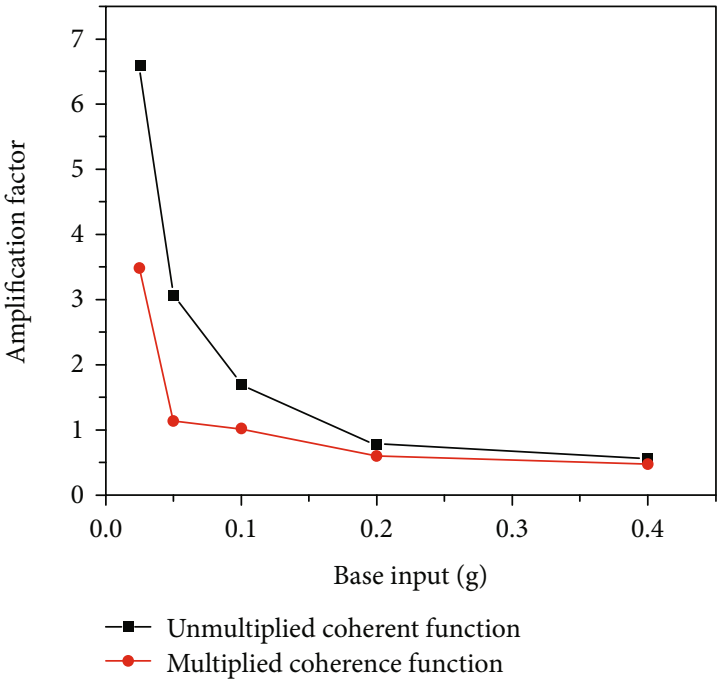

(b)

Figure 10: Amplification factor comparison chart of the unmultiplied coherent function and multiplied coherent function for different conditions (model 2 for the surface with water): (a) first-order mode and (b) second-order mode.

function was small, and the biggest difference was only $0.09 \mathrm{~Hz}$ for the loading of $0.2 \mathrm{~g}$. It can be seen that the influence of the coherence function on the frequency of the second-order mode was less than that of the first-order mode.

It can be seen from Figure 8 that there was a similar situation between the water model and the model without water. The amplification effect of the ground surface mainly occurred in the low frequency stage less than $0.5 \mathrm{~Hz}$. Additionally, at high frequencies above $7 \mathrm{~Hz}$, the amplification factor tended towards zero. As the input load increased, the site response amplification factor decreased. The difference was that the higher-order mode was more obvious in the water model. Furthermore, as shown in Figure 8(e), for the $0.4 \mathrm{~g}$ loading, the amplification factor of the third-order mode of the multiplied coherence function was greater than the amplification factor of the first-order mode shape. In combination with Figure 8 and Table 2, it could be seen that the amplification factor of the unmultiplied coherence function was larger than that of the multiplied coherence function, the amplification coefficient of the first-order mode was greatly different, and that of the second-order and higher-order mode was less different. For example, for the $0.025 \mathrm{~g}$ loading, the amplification factor of the first-order mode of the unmultiplied coherent function was 9.20, while the amplification factor of the first-order mode of the multiplied coherent function was 5.58, with a difference of 3.62. The amplification factor of the second-order mode of the unmultiplied coherent function was 6.58 , and that of the 
second-order mode of the multiplied coherent function was 3.48 , with a difference of 3.1. In the water model, there was a slight difference between the frequency of the first-order mode of the unmultiplied coherent function and that of the multiplied coherent function. The biggest difference was that for the load of $0.025 \mathrm{~g}$, the frequency of the firstorder mode of the unmultiplied coherent function was $0.21 \mathrm{~Hz}$, and the frequency of the first-order mode of the multiplied coherent function was $0.33 \mathrm{~Hz}$. The difference was only $0.12 \mathrm{~Hz}$. The frequency difference of the secondorder mode was negligible.

It can be seen from Figure 9 that for the model without water, the amplification factor of the first- and secondorder modes of both the unmultiplied and multiplied coherent functions decreased with the increase of the input loading. Moreover, the difference between the first mode amplification factor of the unmultiplied coherent function and the multiplied coherent function decreased with the increase of the input loading. Similarly, the difference of the amplification factor between the second-order modes of the unmultiplied coherent function and the multiplied coherent function also decreased with the increase of the input loading, and the difference between the two modes could be ignored when loading $0.4 \mathrm{~g}$. In the water model (as shown in Figure 10), the difference between the unmultiplied and multiplied coherent functions was similar to that in the anhydrous model.

\section{Conclusions}

In this study, the model test of a centrifuge shaking table was used to design two groups of free site models with no water and with water. With the ground motion of different intensities as the base input, the amplified spectrum of the site response was calculated with the traditional spectral ratio method. The influence of the coherent function on the ground motion amplification was analyzed, and the following conclusions were obtained initially.

(1) For different input loading, the site response amplification factor of the unmultiplied coherent function and that of the multiplied coherent function was different from each other, both with a water model and without a water model. In the low frequency stage, the amplification factor of site response multiplied by the coherence function decreased significantly. In the high frequency phase, this downward trend decreased

(2) In these two model tests, the first mode shape of the site response without the multiplication of the coherent function was not visible, and the multiplication of the coherent function reduced the amplification factor and gave rise to an obvious first-order mode. The coherent function had a great influence on the first-order mode of the site response, but the influence on the second-order mode and the higherorder mode was much less than that on the firstorder mode
(3) The model without water had only a single soil layer, which could be regarded as a single degree of the freedom system. However, due to the effect of the surface covering water, the site response of the water model had a relatively obvious high-order mode, which could be regarded as a multidegree of the freedom system. With the increase of the base input, the amplification factor of the high-order mode was higher than that of the first-order mode

\section{Data Availability}

The data used to support the findings and results of this study are available from the corresponding author upon request.

\section{Conflicts of Interest}

The authors declare that they have no conflict of interest.

\section{Acknowledgments}

This research was supported by the National Natural Science Foundation of China (Grant No. 51408559), Guangxi Natural Science Foundation (Grant No. 2018GXNSFAA281183), and Open Fund of the Guangxi Key Laboratory of Geotechnical Mechanics and Engineering (Grant No. 19-Y-21-4). We also thank LetPub (http://www.letpub.com/) for its linguistic assistance during the preparation of this manuscript.

\section{References}

[1] H. P. Ding, X. Jin, Q. F. Liu, and Y. F. Yuan, "Effect of site condition on coherency function of ground motion," Earthquake Engineering and Engineering Vibration, vol. 23, no. 1, pp. 15, 2003.

[2] F. Zheng and J. H. Ye, "Coherency of spatial ground motion field," Journal of Vibration and Shock, vol. 28, no. 6, pp. 2328, 2010.

[3] Y. Chen and J. Li, "Numerical analysis of nonlinear ground motion coherency function of sites under non-consistent excitations," World Earthquake Engineering, vol. 23, no. 4, pp. 7278, 2007.

[4] H. P. Ding and Z. X. Song, "The influence of source parameters on the coherence functions of ground motion," Journal of Vibration and Shock, vol. 29, no. 7, pp. 16-19, 2010.

[5] G. X. Wang, Z. Z. Wang, and D. M. Wang, "Coherence variations of different strong ground motion arrays with depth," Journal of Vibration and Shock, vol. 32, no. 17, pp. 118-122, 2013.

[6] N. A. Abrahamson, J. F. Schneider, and J. C. Stepp, "Spatial coherency of shear waves from the Lotung, taiwan large-scale seismic test," Structural Safety, vol. 10, no. 1-3, pp. 145-162, 1991.

[7] J. H. Steidl, A. G. Tumarkin, and R. J. Archuleta, "What is a reference site?," Bulletin of the Seismological Society of America, vol. 86, no. 6, pp. 1733-1748, 1996.

[8] L. F. Bonilla, J. H. Steidl, J. C. Gariel, and R. J. Archuleta, "Borehole response studies at the Garner Valley downhole array, Southern California," Bulletin of the Seismological Society of America, vol. 92, no. 8, pp. 3165-3179, 2002. 
[9] H. Y. Wang, "Study on variation of soil site amplification with depth: a case at Treasure Island geotechnical array, San Francisco bay," Chinese Journal of Geophysics, vol. 57, no. 5, pp. 1498-1509, 2014.

[10] J. Meng, "Earthquake ground motion simulation with frequency-dependent soil properties," Soil Dynamics and Earthquake Engineering, vol. 27, no. 3, pp. 234-241, 2007.

[11] C. He, J. Wang, C. Zhang, and F. Jin, "Simulation of broadband seismic ground motions at dam canyons by using a deterministic numerical approach," Soil Dynamics and Earthquake Engineering, vol. 76, pp. 136-144, 2015.

[12] S. Fan, Y. Shi, C. Liu, and W. Li, "Simulation of spatially varying seafloor ground motions with random seawater layer and complex terrain," Soil Dynamics and Earthquake Engineering, vol. 111, pp. 110-118, 2018.

[13] V. M. Taboada-Urtuzuastegui, G. Martinez-Ramirez, and T. Abdoun, "Centrifuge modeling of seismic behavior of a slope in liquefiable soil," Soil Dynamics and Earthquake Engineering, vol. 22, no. 9-12, pp. 1043-1049, 2002.

[14] U. Cilingir and S. P. Gopal Madabhushi, "A model study on the effects of input motion on the seismic behaviour of tunnels," Soil Dynamics and Earthquake Engineering, vol. 31, no. 3, pp. 452-462, 2011.

[15] Z. Y. Chen and H. Shen, "Dynamic centrifuge tests on isolation mechanism of tunnels subjected to seismic shaking," Tunnelling and Underground Space Technology, vol. 42, pp. 67-77, 2014.

[16] O. El Shafee, T. Abdoun, and M. Zeghal, "Centrifuge modelling and analysis of site liquefaction subjected to biaxial dynamic excitations," Geotechnique, vol. 67, no. 3, pp. 260271, 2017.

[17] R. D. Borcherdt, "Effect of local geology on ground motion near San Francisco Bay," Bulletin of the Seismological Society of America, vol. 60, no. 1, pp. 29-61, 1970.

[18] O. Yorihiko, Shin Jishindo no spectre Kaiseki Nyumon, Seismological Press, Beijing, 2008. 